\title{
Probing the stellar wind geometry in Vela X-1 with infrared interferometry
}

\section{Élodie Choquet ${ }^{1} \dagger$, Pierre Kervella ${ }^{1}$, Jean-Baptiste Le Bouquin ${ }^{2}$, Antoine Mérand ${ }^{3}$, Xavier Haubois ${ }^{4}$, Jean-Philippe Berger ${ }^{3}$, Guy Perrin ${ }^{1}$, Pierre-Olivier Petrucci ${ }^{2}$, Bernard Lazareff ${ }^{2}$, and Rafael Millan-Gabet ${ }^{5}$}

\author{
${ }^{1}$ LESIA, Observatoire de Paris, CNRS, UPMC, Université Paris-Diderot, PSL, \\ Meudon, France \\ ${ }^{2}$ UJF-Grenoble 1/CNRS-INSU, IPAG, UMR 5274, Grenoble, France \\ ${ }^{3}$ European Southern Observatory, Santiago, Chile \\ ${ }^{4}$ Instituto de Astronomia, Geofisica e Ciências Atmosféricas, Universidade de São Paulo, \\ São Paulo, Brazil \\ ${ }^{5}$ California Institute of Technology, NASA Exoplanet Science Institute, Pasadena, USA \\ ${ }^{\dagger}$ email: elodie.choquet@obspm.fr
}

\begin{abstract}
High-mass X-ray Binaries (HMXBs) are keys to study stellar remnants that are otherwise extremely faint and difficult to observe when isolated. Vela X-1 is a well-known eclipsing HMXB composed of a very massive neutron star orbiting a B0.5I supergiant with a period of 9 days. The supergiant wind is the main feeding material for the accreting neutron star, and its properties are of prime interest to understand the physics at stakes in the accretion process.

In order to characterize the geometry and physical properties of the dense wind at a scale of a few stellar radii, we obtained infrared interferometric observations of Vela X-1 in 2010 using the VLTI/AMBER instrument in the $\mathrm{K}$ band $(2.2 \mu \mathrm{m})$, and in 2012 using the VLTI/PIONIER instrument in the $\mathrm{H}$ band $(1.6 \mu \mathrm{m})$.

Although the apparent disk of the supergiant and the orbital separation of the two objects are beyond the present resolution limit of the VLTI, the K-band observations partially resolve the wind envelope on the two longest baselines. We were able to measure the radius of $265 \pm 82 \mathrm{R}_{\odot}$ for the circumstellar wind at a temperature of $1300 \mathrm{~K}$, assuming a distance of $1.9 \mathrm{kpc}$. The H-band observations do not resolve the system, and we were able to set an upper limit of $112 \mathrm{R}_{\odot}$ for the envelope radius at a temperature of $1800 \mathrm{~K}$.
\end{abstract}

Keywords. X-rays: binaries, Vela X-1, techniques: interferometric, high angular resolution

\section{Introduction}

Vela X-1 is a well-known HMXB hosting a massive pulsar embedded in the dense wind of a B0.5Ib supergiant companion of radius $\sim 32 \mathrm{R}_{\odot}$ (Rawls et al. 2011 ). The system is very luminous in X-rays due to the accretion of the supergiant stellar wind by the neutron star. It also shows constant flares and off-states, interpreted as a clumpy wind with cavities and dense blob, inducing a strongly fluctuating accretion activity with the neutron star motion (Fürst et al. 2010). However, although X-ray observations are of particular interest to understand the accretion process in the immediate vicinity of the neutron star, they fail to provide informations on the structure of the feeding wind at a larger scale. 

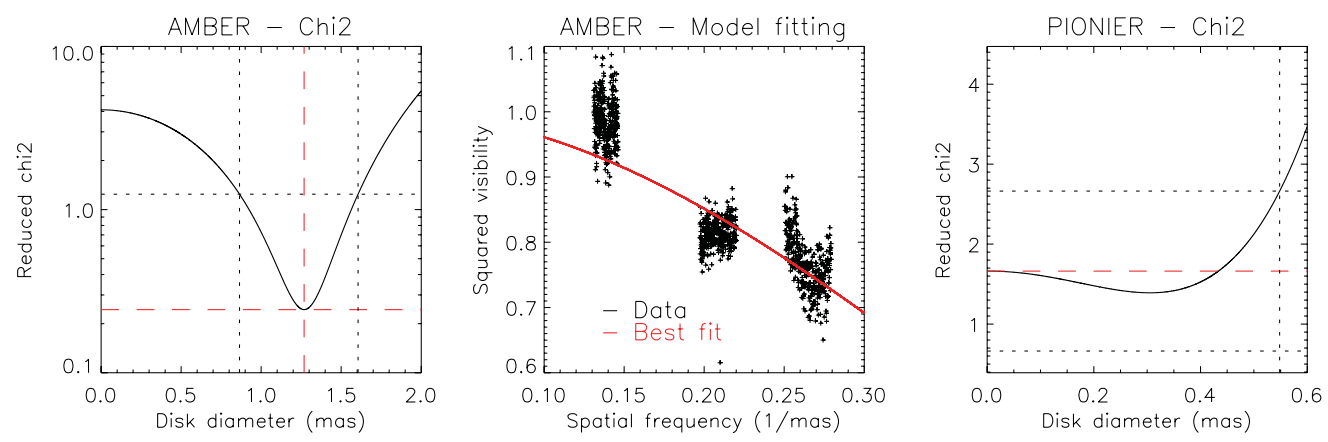

Figure 1. Left: reduced $\chi^{2}$ of the fit of a uniform disk model to the squared visibilities measured in 2010. Middle: squared visibilities measured with the AMBER instrument, and best model of a disk of 1.3 mas. Right: reduced $\chi^{2}$ of the fit of a uniform disk model to the squared visibilities measured in 2012 .

\section{K-band interferometric observations}

We obtained measurements of Vela X-1 on March 2010, using the AMBER instrument at VLTI, combining 3 telescopes (UT1-UT3-UT4) in the K band $(2.2 \mu \mathrm{m})$.

We find that the system is partially resolved on the two longest baselines. We measure an angular diameter of $1.3 \pm 0.4$ mas by fitting the squared visibilities to the model of a uniform disk (see Fig. 1, left and middle). Assuming a distance of $1.9 \mathrm{kpc}$ from Earth, we thus measure a radius of $265 \pm 82 \mathrm{R}_{\odot}$ for the stellar wind of the supergiant.

\section{H-band interferometric observations}

We obtained additional measurements of Vela X-1 in March 2012 using the PIONIER instrument at VLTI, combining 4 telescopes (on A1-G1-I1-K0) in the $\mathrm{H}$ spectral band $(1.6 \mu \mathrm{m})$.We obtained 4 measurement epochs spread over the 9-day period of the binary.

We find that the system is not resolved in this spectral band, for the 4 sampled orbital phases. We constrained the angular diameter by fitting the squared visibilities of the 4 nights to the model of a uniform disk, and deduced an upper limit of 0.55 mas (see Fig. 1, right). Considering a distance of $1.9 \mathrm{kpc}$ from Earth, we thus measured an upper limit of $112 \mathrm{R}_{\odot}$ for the radius of the stellar wind of the supergiant in the $\mathrm{H}$ band.

\section{Conclusion}

Although the upper bound in the $\mathrm{H}$ band corresponds to the diameter expected for this supergiant (Kervella et al. 2004), the important diameter of the wind at $1300 \mathrm{~K}$ is unexpected. We deduced two possible explanations for this strong difference.

- we resolved a diffuse shell in 2010 due to a transient event, that was not observable any longer in 2012;

- there is a strong temperature gradient in the dense stellar wind, and the hot material at $1800 \mathrm{~K}$ is more compact than the material at $1300 \mathrm{~K}$.

\section{References}

Fürst, F., Kreykenbohm, I., Pottschmidt, K., Wilms, J., et al. 2010, A 6 A, 519, A37

Rawls, M. L., Orosz, J. A., McClintock, J. E., Torres, M. A. P., et al. 2011, ApJ, 730, 25

Kervella, P., Thévenin, F., Di Folco, E., \& Ségransan, D. 2004, A\&A, 426, 297 